\title{
Clinical Evaluation of Hepatic Fibrosis in Chinese Patients with Nonalcoholic Fatty Liver Disease with FibroScan
}

\author{
Zeli Gao ${ }^{*}$, Xiaoli Lin ${ }^{2}$, Guoyu Chen' ${ }^{1}$ Jieru Ji1 ${ }^{1}$, Mingfeng Tang2 ${ }^{2}$ Xiaotong He1, \\ Qinghui Xiong2, Ming Li ${ }^{2 *}$ \\ ${ }^{1}$ Department of Gastroenterology, People's Hospital of Pudong New Area, Shanghai, China \\ ${ }^{2}$ Laboratory of Innovative Medicine, Hong Kong \\ Email: ${ }^{*}$ gzeli@163.com, ${ }^{*}$ liming@labinnovativem
}

Received 27 March 2015; accepted 12 May 2015; accepted 15 May 2015

Copyright (C) 2015 by authors and Scientific Research Publishing Inc.

This work is licensed under the Creative Commons Attribution International License (CC BY). http://creativecommons.org/licenses/by/4.0/

\section{(c) (i) Open Access}

\begin{abstract}
Aim: The clinical value of FibroScan in evaluation of hepatic fibrosis was assessed in Chinese patients with nonalcoholic fatty liver disease (NAFLD). Methods: Liver stiffness and other NAFLD related variables were measured in NAFLD patients with either elevated or normal hyaluronic acid (HA) levels, and in healthy volunteers respectively. Moreover, hepatic fibrosis rat model was used for the correlation analysis between the measured liver elasticity and its corresponding pathological changes. Results: The severity of NAFLD in patients was positively correlated with both HA level and FibroScan values suggesting that fibrosis occured along with the progression of NAFLD. Interestingly, the FibroScan value was found to change at an earlier stage of the disease than that of HA level. The mean liver elasticity in the sampling volume of the region of interest in experimental rats, which were subject to the induction of liver fibrosis using peritoneal injection of carbon tetrachloride (CCl4) for 6 or 9 weeks respectively, was measured using Fibroscan. The examination of pathological changes of these experimental rats demonstrated the positive correlation of the measured elasticity values with the extent (severity) of the corresponding pathological changes in the rats of 6 weeks post induction of liver fibrosis with a moderate liver fibrosis, or 9 weeks with a severe liver fibrosis. The average liver stiffness for the normal liver (S0) was $4.51 \pm 0.82 \mathrm{kPa}$, for moderate liver fibrosis (S2) was $6.91 \pm 1.32 \mathrm{kPa}$, and for severe liver fibrosis (S3) $9.62 \pm 1.82 \mathrm{kPa}$. Conclusion: FibroScan can be used to objectively and quantitatively identify the trend in the changing stiffness of the liver and noninvasively detect the development of liver fibrosis.
\end{abstract}

\footnotetext{
${ }^{*}$ Corresponding author.
} 
Keywords

FibroScan, Nonalcoholic Fatty Liver Disease, Hyaluronic Acid, Ultrasound Elastography

\section{Introduction}

The incidence of fatty liver disease is gradually increasing and has become the second most severe type of liver disease after viral hepatitis due to the changing of lifestyle in worldwide population [1] [2]. Without proper treatment, fatty liver disease gradually develops into inflammatory cell infiltration and necrosis eventually resulting in liver fibrosis and cirrhosis [3]. Histopathological examination via an invasive liver puncture biopsy has been considered as the gold standard for evaluation of the severity of fatty liver disease [4]-[6]. However, the invasive liver puncturing and its associated risks and complications are not readily accepted by patients. In addition, hepatic fibrosis is a dynamic process, and monitoring the entire process of fibrosis development via a single puncture biopsy is not only difficult, but also not reliable for a representative reflection of the condition of the entire liver [7] [8]. Therefore, an appropriate imaging examination is preferred for not only a non-invasive, but also a dynamic evaluation of the severity and development of fatty liver disease.

Non-invasive transient elastography (FibroScan) is an emerging credible alternative to the invasive biopsy procedure. The FibroScan (Echosens, Paris, France) is a novel rapid, non-invasive, and reproducible method for evaluation of liver stiffness. The ultrasound elastography method can objectively and quantitatively demonstrate elasticity information of tissue and reflect the stiffness of the measured tissue using grayscale or color images [9]. Tissue stiffness is directly proportional to the square of shear wave velocity: the stiffer the tissue is, the faster the shear wave propagates [10]. The present study was designed to investigate the clinical value of FibroScan in evaluating hepatic fibrosis in Chinese patients with NAFLD. Furthermore, whether the elasticity of the liver measured using FibroScan was positively correlated with the degree of pathological change in liver fibrosis was also investigated in animal model.

\section{Methods}

\subsection{Patient Recruitment}

In this study, we included 59 patients with NAFLD aged 20 - 50 years, who had sought medical treatment in our hospital either on an inpatient or outpatient basis in the Department of Gastroenterology between July 2010 and July 2011. Thirty volunteers screened in the Physical Examination Center of the hospital were included in the control group. NAFLD was diagnosed in accordance with the criteria specified by the Fatty Liver and Alcoholic Liver Disease Group of the Chinese Association of Medicine [11]. The liver function of all recruited individuals was within the normal range, and body mass index (BMI) was $<30 \mathrm{~kg} / \mathrm{m}^{2}$. Individuals with a history of longterm alcohol abuse, viral hepatitis, diabetes, hypertension, and systemic sclerotic diseases were excluded from the study. The protocols in this study were approved by Hospital Medical Ethics Committee, People's Hospital of Pudong New Area, China (Approval ID: PRH 09088). Written informed consent was obtained from every patient.

Group A included 30 volunteers (16 men and 14 women) with a mean age of $32.08 \pm 5.96$ years. The patients with NAFLD were assigned to 2 groups depending on their hyaluronic acid (HA) levels. group B: Patients with normal HA levels (16 men and 14 women; mean age, $30.23 \pm 8.68$ years), with elevated HA levels (15 men and 14 women; mean age, $36.74 \pm 6.88$ years) were assigned to group $C$, respectively. There was no significant difference in age and sex between the 3 groups.

\subsection{Evaluation of NAFLD Related Variables of Fibrosis \& Elastography}

NAFLD related variables of the patients, including total bilirubin (TB), aspartate transaminase (AST), alanine transaminase (ALT), gamma-glutamyl transferase (GGT), alkaline phosphatase (ALP), and total bile acid (TBA) levels, were determined using the LX-20 automated biochemical analyzer (Beckerman, USA). HA levels were detected by radioimmunoassay. Body mass index (BMI) was calculated by measuring weight and height. Ab- 
dominal computer tomography (CT) scan was taken to calculate the liver/spleen (L/S) CT value.

FibroScan (Echosens, Paris, France) was performed according to the manufacturer's manual [12] [13]. The scanning points were selected at the intercostal space between 7 and 8 or between 8 and 9 along the right anterior axillary to the abdominal midline. Effective scanning was performed 10 times consecutively, and the median value was used as the final value of the measurement, expressed as $k P a$. The final success rate was required to be $>50 \%$, and an interquartile range of lower than one-third of the measured value was considered reliable.

\subsection{Animal Model}

In order to avoid the invasive procedure of liver puncture biopsy in human patients and to demonstrate the close correlation of the measured liver elasticity with the corresponding pathological changes, liver fibrosis model was induced in male Wistar rats $(160-180 \mathrm{~g}, \mathrm{n}=30)$ by intraperitoneal injection of Carbon tetrachloride $(50 \%$ dissolved in plant oil, $3 \mathrm{ml} / \mathrm{kg}$ body weight/time) twice a week for $6(\mathrm{n}=15)$ or 9 weeks $(\mathrm{n}=15)$ to develop liver fibrosis. Another 10 male Wistar rats (160 - $180 \mathrm{~g})$ were served as normal reference control.

\subsection{FibroScan $®$ Evaluation}

FibroScan was used for the evaluation of liver stiffness. At the time points prior to the induction of liver fibrosis (LF), 6 and 9 weeks post induction of the LF, the stiffness of the liver in these experimental animals was determined. The rats were fixed in the supine position with anesthesia. The skin was prepared from the fourth right rib to the abdomen so as to fully expose the area to be examined. The probe was gently moved without exerting any pressure and once the image was stable, the frame was determined. The elasticity in the selected region of interest was measured using the method provided by the ultrasonic apparatus. For sampling, two segments of the hepatic right lobe in the longitudinal section and one segment of the left lobe in the xiphoideusal horizontal section were selected; three samples were taken from each segment. When the deviation of the values determined for the three samples was $<10 \%$, the sampling was considered to be successful. Mean values were calculated for each segment, and the mean value of the means of all the segments was calculated to obtain the required data. Data determined for each group were the maximum elastic modulus (Max), the mean elastic modulus (Mean), the minimum elastic modulus (Min). Max reflected tissue structures with the highest stiffness within a sampling area, including diseased tissue and certain structures in the liver, such as ligaments, whereas Min reflected tissue structures with the lowest stiffness in a sampling area, including the lumens of the intrahepatic blood vessels and the bile duct. Mean was the mean elastic modulus of the entire area and the standard deviation reflected the uniformity of the tissue stiffness in the sampling area, and the successful sampling rate. The results were expressed in kilopascals $(\mathrm{kPa})$. The median value was considered as representative of the elastic modulus of the liver.

\subsection{Morphological Evaluation}

The experimental animals were sacrificed after the final elastography measurements on week 6 or week 9 respectively using $\mathrm{CO}_{2}$ inhalation performed in compliance with AVMA panel on euthanasia. Briefly, the animal holding chamber was charged slowly with $\mathrm{CO}_{2}$ to a concentration of $70 \%$ from a compressed $\mathrm{CO}_{2}$ gas in cylinder. The gas flow was maintained for at least $1.5 \mathrm{~min}$ after apparent clinical death was verified. The liver specimen were harvested and washed with PBS. For histological and immunohistochemical examination, the specimen were cut into appropriate sizes and fixed with $10 \%$ formalin, followed by routine dehydration, vitrification, waxing, embedding. The paraffin block containing specimen slices were sectioned $(5 \mu \mathrm{m})$. The thin sections were stained with hematoxylin and eosin ( $\mathrm{H} \& \mathrm{E}$ ) or Masson's trichrome according to kit directions, which labels the fibrous collagen blue and liver cells red. The blue stained areas were digitized and quantified morphometrically.

The severity of pathological changes of hepatic steatosis in the experimental animals was determined under a light microscope using a five degree classes according to the area of steatosis that was observed in the liver cell specimen [14] [15]. Normal liver F0, steatosis area $<10 \%$; slight steatosis F1, steatosis area $10 \%$ - 33\%; mild fatty liver F2, steatosis area $33 \%-50 \%$; moderate fatty liver F3, steatosis area $50 \%-66 \%$; and severe fatty liver $\mathrm{F} 4,>66 \%$ steatosis of liver cells.

The staging of pathological changes of hepatic fibrosis in the experimental animals was morphometrically calculated under microscope according to the formation of fibrous tissue in the liver cell specimen [16]. Stage 
S0, normal liver without fibrosis surrounding the portal triads (the groupings of portal veins, hepatic arteries, and bile ducts in the liver lobules); S1, fibrous connective tissue formed in a small region, predominantly surrounding the portal triads but is limited to these areas; S2, fibrous tissues begin to extend into the periportal space forming thin fibrous septa, but do not connect portal area to any other with the lobular structure being roughly retained; S3, fibrous tissues forming a large quantity of fibrous septa, which link neighboring portal triads and extend to the central veins causing the lobular structure to be disordered but without cirrhosis; S4, early cirrhosis. Most portal areas are connected by fibrous tissue, and some portal areas and central veins are also connected. Hepatocyte clusters are now completely surrounded by fibrous tissue, producing cirrhotic nodules.

\subsection{Statistical Analysis}

Statistical analysis was performed using SPSS 15.0. Data were expressed as mean \pm SD. Analysis of variance was used for comparing the mean values between $>2$ groups. Inter-group comparison was performed by Student $t$ test.

\section{Results}

Clinical examination results of human subjects showed that no significant difference in liver function or BMI between the 3 groups were found $(P>0.05$, Table 1). Although there was no significant difference in HA levels between groups $\mathrm{A}$ and $\mathrm{B}$, the $\mathrm{HA}$ level of patients in group $\mathrm{C}$ was significantly higher than those in $\mathrm{A}$ and $\mathrm{B}$ $(P<0.01)$. Interestingly, the patients in group $\mathrm{C}$ displayed the lowest $\mathrm{L} / \mathrm{S} \mathrm{CT}$ value compared with those in patients of group B (median) and healthy subjects of group A (lowest) $(P<0.05)$. More interestingly, there is significant differences in FibroScan values between the 3 groups $(P<0.05)$, this value being the highest in group $\mathrm{C}$ and the lowest in group A, indicating that the extents of stiffness of the liver among the three groups are $\mathrm{C}>\mathrm{B}>$ A. Paired comparison between the groups showed a significant difference $(P<0.05)$. The details of comparison of HA levels, L/S CT values, and FibroScan values are shown in Table 2. The clue of these results suggested that FibroScan is a more sensitive method to monitoring the progress of liver fibrosis than HA level changes, which is supported by the L/S CT result in this study.

To test the correlation of hepatic elasticity evaluation with the histopathological changes, FibroScan ${ }^{\circledR}$ evaluation of hepatic steatosis and fibrosis in animal model was conducted. Although the hair of the experimental rats at 6 weeks post LF induction appears almost normal with little hair loss, the experimental animals at 9 weeks post LF induction experienced a progressive hair loss during the last 3 weeks of the experiment. Approximately

Table 1. Comparison of live function and BMI among the three groups.

\begin{tabular}{cccccc}
\hline Parameter & Group A & Group B & Group C & F value & $P$ value \\
\hline TB $(\mathrm{mmol} / \mathrm{L})$ & $8.33 \pm 2.99$ & $8.86 \pm 3.59$ & $8.75 \pm 3.20$ & 0.104 & 0.902 \\
ALT $(\mathrm{mmol} / \mathrm{L})$ & $30.27 \pm 5.62$ & $34.29 \pm 5.36$ & $32.58 \pm 5.40$ & 1.975 & 0.153 \\
AST $(\mathrm{mmol} / \mathrm{L})$ & $28.47 \pm 9.06$ & $26.14 \pm 8.73$ & $30.08 \pm 7.39$ & 0.712 & 0.497 \\
GGT $(\mathrm{mmol} / \mathrm{L})$ & $78.86 \pm 17.30$ & $76.07 \pm 25.61$ & $73.92 \pm 23.62$ & 0.168 & 0.846 \\
ALP $(\mathrm{mmol} / \mathrm{L})$ & $86.60 \pm 12.56$ & $77.71 \pm 15.18$ & $84.00 \pm 13.90$ & 1.545 & 0.226 \\
TBA $(\mathrm{mmol} / \mathrm{L})$ & $13.19 \pm 1.35$ & $14.06 \pm 1.84$ & $13.61 \pm 1.64$ & 2.133 & 0.156 \\
BMI $\left(\mathrm{kg} / \mathrm{m}^{2}\right)$ & $22.80 \pm 2.88$ & $23.36 \pm 2.37$ & $24.42 \pm 2.11$ & 1.407 & 0.257
\end{tabular}

Table 2. Comparison of HA, CT value and FS value among the three groups $(\mathrm{x} \pm \mathrm{s})$.

\begin{tabular}{ccccc}
\hline Parameters & Group A & Group B & Group C & F value \\
\hline HA (ug/L) & $95.07 \pm 17.38$ & $93.00 \pm 19.79$ & $133.42 \pm 8.12^{\Delta \triangleleft}$ & 24.923 \\
L/S CT value & $1.06 \pm 0.07$ & $0.72 \pm 0.10^{*}$ & $0.43 \pm 0.04^{\# *}$ & 272.25 \\
FS value $(k P a)$ & $3.54 \pm 0.66$ & $5.65 \pm 0.76^{\Delta}$ & $6.83 \pm 0.99^{\Delta \#}$ & 58.924 \\
\hline
\end{tabular}

Note: compared with group $\mathrm{A},{ }^{*} P<0.05,{ }^{\Delta} P<0.01$; compared with group $\mathrm{B},{ }^{\sharp} P<0.05,{ }^{\circ} P<0.01$. 
$60 \%$ of the rats at 9 weeks post induction of LF exhibited a smaller liver with an increased echo. Most of the experimental rats with smaller liver presented with ascites, which were indicated by a dark area of $0.5-1 \mathrm{~cm}$ diameter in the space between the liver and the kidney.

Liver elasticity was measured using FibroScan and the mean liver elasticity in the sampling volume of the region of interest in the liver were approximately $4.51 \pm 0.82$ in the normal reference control rats and in the rats of test group prior to the induction of LF by intra-peritoneal injection of carbon tetrachloride. However, the average elasticity values increased to $6.91 \pm 1.32$ and $9.62 \pm 1.82$ in the experimental rats of test group at 6 and 9 weeks post induction of the LF. These results indicated that intraperitoneal injection of Carbon tetrachloride might have induced damages to the liver leading to hepatic steatosis and progressive fibrosis, which progressively increased the stiffness of the liver, and hence the progressively increased elasticity of the liver.

After the second FibroScan at 6 weeks post induction of LF, 15 animals of the test group were sacrificed and the remaining 15 rats were sacrificed at 9 weeks after the last FibroScan. When the abdomen of the rats of test group sacrificed at 9 weeks was opened, marked ascites was observed in $60 \%$ of the experimental animals with the size of spleen significantly increased due to liver fibrosis and cirrhosis. The size of liver remarkably decreased due to cirrhosis and the liver surface appeared coarse granular. By contrast, no ascites was observed and the size and appearance of liver were normal without splenomegaly observed in animals of normal reference control group.

In order to demonstrate whether the increased elasticity of the liver measured by FibroScan is closely and positively correlated with the pathological changes of the liver, the pathological studies with the liver specimen of the experimental animals were carried out. The pathological observation showed that the hepatic parenchymal cells of rats in normal reference control group appeared normal and intact with few lipid droplet or vacuoles observed, and the lobules and the hepatic cords normally completely structured (Figure 1: NRC). By comparison, lipid droplets or vacuoles were scattered in the liver cells of the rats in test group. These lipid droplets may have fused and undergone nuclear deviation or centered around the nucleus. The areas of steatosis accounted for $40 \%$ of the total area of the liver at 6 weeks, which was classified as mild fatty liver F2, or 70\% at 9 weeks, which was identified as severe fatty liver F4. The intracellular structure of the liver cells at stage of F4 was not able to be identified.

Masson trichrome staining indicated the following: There was no evident fibrosis surrounding the portal triads with normal liver cell and lobular structure observed in the livers of the rats in the normal reference control group (in average: S0) (Figure 1: NRC). By contrast, the blue-stained thin fibrous collagen expansion formed extending into the periportal space forming thin fibrous septa with the lobular structure being roughly retained at 6 weeks post induction of LF (in average: S2) (Figure 2: 6W). However, relatively thicker blue-stained fibrous collagen expansion formed marked bridging between portal to portal (p-p) and portal to central (p-c) regions in the whole overall image forming marked and significantly smaller pseudolobule, which covers a considerable area $(60 \%-80 \%)$ of the liver 9 weeks post LF induction (Figure 3: 9W). Hepatocyte clusters, which are completely surrounded by thick fibrous partitions producing cirrhotic nodules, were found throughout the whole area of the liver (in average: S3-4).

The correlation analysis indicated that the mean elasticity of the liver of rats measured by FibroScan in both groups was positively correlated with the pathological steatosis area $(P<0.01)$. Furthermore, the analysis of variance results of the experimental rats in both groups at the different fibrosis stages (S0-4) indicated statistically significant differences among the mean elasticity values for the different stages. The mean elasticity values were approximately as follows: S0, $4.1-5.5 \mathrm{kPa}$; $\mathrm{S} 1,4.8$ - $5.8 \mathrm{kPa}$; S2, 6.4 - $6.8 \mathrm{kPa}$; S3, 6.1 - $11.6 \mathrm{kPa}$; and S4, 10.6 $16.4 \mathrm{kPa}$. The histopathological results indicated that a higher stage of fibrosis implied a greater elastic modulus (stiffness). Liver stiffness values were positively correlated with stages of liver fibrosis $(P<0.05)$. Therefore, FibroScan can be reliably used to distinguish patients with no or mild liver fibrosis from those with moderate or advanced fibrosis in NAFLD.

\section{Discussion}

Comprehensive efforts have been made in recent years to explore noninvasive techniques for the assessment of hepatic fibrosis. Some clinical, biochemical, and imaging indexes have also been found useful for assessing the extent of hepatic fibrosis. Liver elastography (hardness measurement) is one of such noninvasive technique for diagnosing hepatic fibrosis. In 2000, Sanada et al. reported an ultrasound imaging system and reconstructed a 


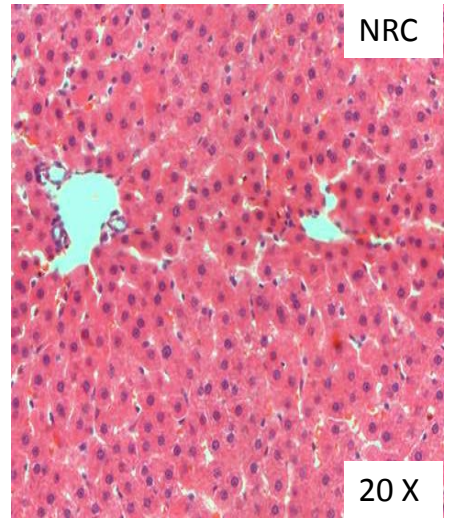

Figure 1. The pathological analysis of the experimental rats. The representative phenotype and structures of the parenchymal cell, lobule and portal cord of the liver in normal reference control rats (NRC) appeared complete, normal and intact with almost no lipid droplet (F0) or blue-stained fibrous septa (S0) observed.

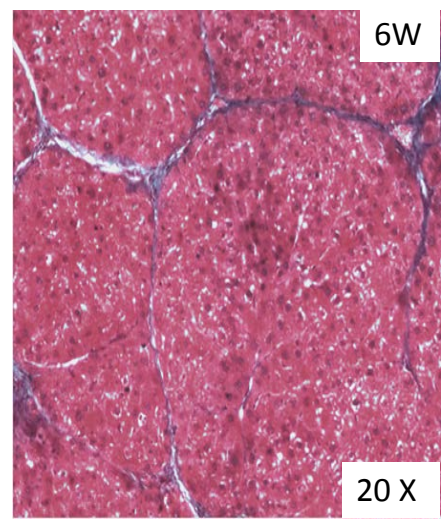

Figure 2. Lipid droplets were found in the liver cells of the rats sacrificed at 6 weeks post induction of LF (6W), which accounts for approximately $40 \%$ area (F2) of the entire liver area. Blue-stained thin fibrous collagen expansion was observed extending into the periportal space forming thin fibrous septa with the lobular structure being roughly retained (S2).

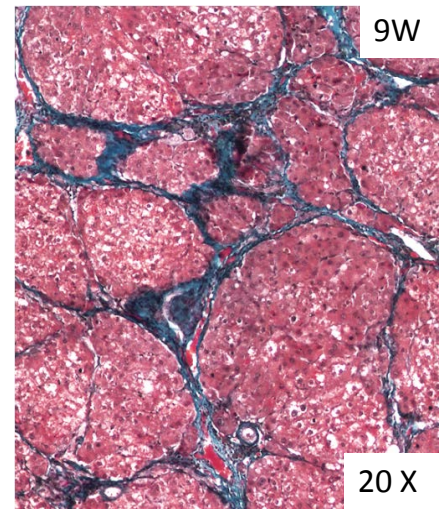

Figure 3. Thick blue-stained fibrous collagen expansion formed networks between portal to portal ( $\mathrm{p}-\mathrm{p})$ and portal to central (p-c) regions in the entire liver, which covers approximately $70 \%$ area of the entire liver (F4) 9 weeks post LF induction (9W). Hepatocyte clusters completely surrounded by blue-stained fibrous septa producing cirrhotic nodules, which were found throughout the whole area of the liver (S3-4). 
vibration spectrum of the soft tissue under forced mechanical vibration [17]. Vibration rate is used as an index to evaluate the characteristics of elastic shear of the soft tissue in both healthy subjects and patients with chronic hepatitis and cirrhosis. It was found that the vibration rate is significantly and positively correlated with the extent of hepatic fibrosis formation. Thus, this technique could be used to objectively determine the degree of hepatic fibrosis [18]. Since patients with hepatic fibrosis and cirrhosis have high elasticity values, thus, FibroScan can be used as a non-invasive alternative to replace invasive liver puncture biopsy for liver histological assessment [19]. In addition, some studies have also evaluated the success rate and accuracy of FibroScan. A study involving 2114 patients with chronic hepatitis failed to obtain sufficient signal echo in 96 patients. Multivariate analysis showed that the failure to obtain sufficient signal echo was mainly affected by the BMI of the patients $\left(\mathrm{BMI}>28 \mathrm{~kg} / \mathrm{m}^{2}\right)$ and less affected by the factors, such as the operator, sex, or ALT levels. The reason for this is probably due to the ultrasound decays rapidly in fat tissue [8]. However, other report found that there is strong correlation between liver stiffness measurement and insulin resistance index, but medium correlation with proportion of excess of fat in body composition in NAFLD patients [20].

Thus, this technique is now mainly used for the evaluation of cirrhosis in patients with chronic hepatitis $\mathrm{C}$ and alcoholic liver disease [21]. The application of this technique in assessment of NAFLD-induced hepatic fibrosis remains controversial [22] since liver puncture biopsy and FibroScan may deliver different results in particular in the obese population [23]. Hepatic fibrosis is a process of diffuse and excessive deposition of extracellular matrix in the liver that is a consequence of excessive deposition or degradation of the extracellular matrix [24]. HA is a component of the matrix that is synthesized by mesenchymal cells, and therefore, HA levels can be a sensitive and accurate biochemical indicator of the extent of fiber formation in the liver and the degree of impairment of hepatocytes. Some researchers believe that HA levels can provide the entire liver profile compared with liver puncture biopsy. Hence, HA level can be used as a sensitive indicator for hepatic fibrosis and cirrhosis [25].

In this study, we consistently confirmed the positive correlation of the severity of NAFLD in Chinese patients with elevation of HA levels. More importantly, we not only demonstrated that FibroScan values were positively correlated with the severity of NAFLD in Chinese NAFLD patients, but also found changes in FibroScan values occurred earlier than elevation of HA levels, implying that fibrosis occurred along with the progression of NAFLD. With the continuous improvement in living standard of Chinese population, the prevalence of obesity, diabetes, and hyperlipidemia has steadily increased that in turn, has resulted in an increase in the number of patients with NAFLD [26], the serious consequence of which is the progression of NAFLD to hepatic fibrosis, cirrhosis, and end-stage liver disease [27]. Since NAFLD is a reversible disease at its early stage, therefore, early diagnosis of the disease is of particular importance. Although liver puncture biopsy is the gold standard for the assessment of NAFLD and its consequent hepatic fibrosis and cirrhosis, this invasive procedure has been reported to associate with pain [28], or life-threatening complications [29]. Furthermore, since the development of NAFLD to liver fibrosis and cirrhosis is a dynamic process, therefore, the invasive nature associated with liver puncture biopsy makes tracking the progression of the condition in a time course and multi-regions of the liver is almost impossible. In addition, the clinical application of liver puncture biopsy is also limited by the technical availability of the technique in some local hospitals and medical care facilities. By comparison, since the non-invasive nature, the good reproducibility, and dynamic observation of FibroScan, it can be easily used for tracking the progression of the condition in a time course and multi-regions of the liver. So that early detection and diagnosis of NAFLD is possible by application of ultrasound elastography in screening of patients with NAFLD in order to prevent its progression to fibrosis and cirrhosis within the reversible treatment window.

In order to determine the correlation between the ultrasonography and the pathological changes, all of the experimental animals underwent ultrasonography, which was correlatively compared with the pathological changes of each particular experimental animal. Following H\&E staining, the lipid droplets observed in the liver cells were morphometrically analyzed. The fatty liver was categorized into stages F0-4 according to the proportion of liver cells that exhibited steatosis in the liver tissue sections. Masson's trichrome staining labels the fibrous collagen blue and liver cells red, which enables clear observation of the fibrous tissue in the liver for qualitative or quantitative diagnosis of liver fibrosis. Liver fibrosis is divided into stages S1-4 according to the deposition site and scope of the collagen fibers, the damage to the liver structure and its influence on the hepatic microcirculation.

Although the data and analysis of present study indicated that the hepatic steatosis area and the fibrosis grade positively correlated with the elastic modulus of the liver, we do agree with the notion that the fibrosis may be 
the key element contributing to liver stiffness [30]. The development of fatty liver disease proceeds from a simple fatty liver to steatohepatitis, fibrosis and liver cirrhosis [31] [32]. The reason for the increased elasticity value of the liver with higher grade of hepatic steatosis is probably due to the coexisting of steanosis with fibrosis in a particular liver at the time of FibroScan measurement, which can be confirmed by the observation of the histological sections, in which the liver cell steatosis and hepatic fibrosis coexists. Furthermore, the present study also showed that the hepatic steatosis area and liver stiffness were positively associated with the severity of fibrosis, identifying a positive correlation between them.

\section{Conclusion}

We have demonstrated the positive correlation of the severity of NAFLD in Chinese patients with the elevation of HA levels. More importantly, we found that changes in FibroScan values occurred earlier than the elevation of HA levels in Chinese NAFLD patients. Furthermore, we have demonstrated the positive correlation of liver elastography with the severity of pathological changes in fatty liver and liver fibrosis animal model. These significant results should shed light to clinical application of FibroScan for early diagnosis of NAFLD so as to prevent its progression to hepatic fibrosis and cirrhosis within its reversible treatment window.

\section{Acknowledgements}

This study was supported by Key Disciplines Group Construction Project of Pudong Health Bureau of Shanghai (Grant No. PWZxq2014-13).

\section{References}

[1] Adams, L.A. and Angulo, P. (2006) Treatment of Non-Alcoholic Fatty Liver Disease. Postgraduate Medical Journal, 82, 315-322. http://dx.doi.org/10.1136/pgmj.2005.042200

[2] Paschos, P. and Paletas, K. (2009) Non Alcoholic Fatty Liver Disease and Metabolic Syndrome. Hippokratia, 13, 9-19.

[3] Brunt, E.M. (2004) Nonalcohlic Steatohepatitis. Seminars in Liver Disease, 24, 3-20. http://dx.doi.org/10.1055/s-2004-823098

[4] Wahl, K., Rosber, G., Vaske, B., et al. (2012) Biopsy-Controlledliver Fibrosis Staging Using the Enhanced Liver Fibrosis Score Comparedto Transient Elastography. PLoS ONE, 7, e51906.

[5] Scott, D.R. and Levy, M.T. (2010) Liver Transient Elastography (Fibroscan ${ }^{\mathbb{B}}$ ): A Place in the Management Algorithms of Chronic Viral Hepatitis. Antiviral Therapy, 15, 1-11. http://dx.doi.org/10.3851/IMP1474

[6] Kawamoto, M., Mizuguchi, T., Katsuramaki, T., Nagayama, M., Oshima, H., Kawasaki, H., et al. (2006) Assessment of Liver Fibrosis by a Noninvasive Method of Transient Elastography and Biochemical Markers. World Journal of Gastroenterology, 12, 4325-4330.

[7] Alisi, A., Pinzani, M. and Nobili, V. (2009) Diagnostic Power of Fibroscan in Predicting Liver Fibrosis in Nonalcoholic Fatty Liver Disease. Hepatology, 50, 2048-2049. http://dx.doi.org/10.1002/hep.23345

[8] Foucher, J., Castera, L., Bernard, P.H., Adhoute, X., Laharie, D., Bertet, J., et al. (2006) Prevalence and Factors Associated with Failure of Liver Stiffness Measurement Using FibroScan in a Prospective Study of 2114 Examinations. European Journal of Gastroenterology \& Hepatology, 18, 411-412. http://dx.doi.org/10.1097/00042737-200604000-00015

[9] Greenleaf, J.F., Fatemi, M. and Insana, M. (2003) Selected Methods for Imaging Elastic Properties of Biological Tissues. Annual Review of Biomedical Engineering, 5, 57-78. http://dx.doi.org/10.1146/annurev.bioeng.5.040202.121623

[10] Bercoff, J., Tanter, M. and Fink, M. (2004) Supersonic Shear Imaging: A New Technique for Soft Tissue Elasticity Mapping. IEEE Transactions on Ultrasonics Ferroelectrics and Frequency Control, 51, 396-409.

[11] Fatty Liver and Alcoholic Liver Disease Group of the Chinese Association of Medicine (2006) The Criteria for the Diagnosis of NAFLD. Chin J Hepatol, 14, 161-163.

[12] Sandrin, L., Fourquet, B., Hasquenoph, J.M., Yon, S., Fournier, C., Mal, F., et al. (2003) Transient Elastography: A New Noninvasive Method for Assessment of Hepatic Fibrosis. Ultrasound in Medicine \& Biology, 29, 1705-1713. http://dx.doi.org/10.1016/i.ultrasmedbio.2003.07.001

[13] de Ledinghen, V., Wong, V.W. and Vergniol, J. (2012) Diagnosis of Liver Fibrosis and Cirrhosis Using Liver Stiffness Measurement: Comparison between M and XL Prob of Fibroscan. Journal of Hepatology, 56, 833-839. http://dx.doi.org/10.1016/j.jhep.2011.10.017 
[14] Brunt, E.M., Janney, C.G., Di Bisceglie, A.M., Neuschwander-Tetri, B.A. and Bacon, B.R. (1999) Nonalcoholic Steatohepatitis: A Proposal for Grading and Staging the Histological Lesions. The American Journal of Gastroenterology, 94, 2467-2474. http://dx.doi.org/10.1111/j.1572-0241.1999.01377.x

[15] Burt, A.D., Mutton, A. and Day, C.P. (1998) Diagnosis and Interpretation of Steatosis and Steatohepatitis. Seminars in Diagnostic Pathology, 15, 246-258.

[16] Knodell, R.G., Ishak, K.G., Black, W.C, et al. (1981) Formulation and Application of a Numerical Scoring System for Assessing Histological Activity in Asymptomatic Chronic Active Hepatitis. Hepatology, 1, 431-435. http://dx.doi.org/10.1002/hep.1840010511

[17] Sanada, M., Ebara, M., Fukuda, H., Yoshikawa, M., Sugiura, N., Saisho, H., et al. (2000) Clinical Evaluation of Sonoelasticity Measurement in Liver Using Ultrasonic Imaging of Internal Forced Low-Frequency Vibration. Ultrasound in Medicine \& Biology, 26, 1455-1460. http://dx.doi.org/10.1016/S0301-5629(00)00307-0

[18] Kim, K.M., Choi, W.B., Park, S.H., et al. (2007) Diagnosis of Hepatic Steatosis and Fibrosis by Transient Elastography in Asymptomatic Healthy Individuals: A Prospective Study of Living Related Potential Liver Donors. Journal of Gastroenterology, 42, 382-388. http://dx.doi.org/10.1007/s00535-007-2016-1

[19] Poynard, T., de Ledinghen, V., Zarski, J.P., et al. (2012) Relative Performances of FibroTest, Fibroscan, and Biopsy for the Assessment of the Stage of Liver Fibrosis in Patients with Chronic Hepatitis C: A Step Towardthe Truth in the Absence of a Gold Standard. Journal of Hepatology, 156, 541-548.

[20] Topil'skaia, N., Morozov, S., Isakov, V., Trufanova, I. and Kaganov, B. (2011) [Elastography Efficiency in the Liver Fibrosis Determination in Patients with Nonalcoholic Fatty Liver Disease]. Éksperimental'naiâ $i$ Klinicheskaia Gastroenterologiia (Experimental \& Clinical Gastroenterology), 26, 26-31.

[21] Ziol, M., Handra-Luca, A., Kettaneh, A., et al. (2005) Noninvasive Assessment of Liver Fibrosis by Measurement of Stiffness in Patients with Chronic Hepatitis C. Hepatology, 41, 48-54. http://dx.doi.org/10.1002/hep.20506

[22] Kim, K.M., Choi, W.B., Park, S.H., Yu, E., Lee, S.G., Lim, Y.S., et al. (2007) Diagnosis of Hepatic Steatosis and Fibrosis by Transient Elastography in Asymptomatic Healthy Individuals: A Prospective Study of Living Related Potential Liver Donors. Journal of Gastroenterology, 42, 382-388. http://dx.doi.org/10.1007/s00535-007-2016-1

[23] Myers, R.P., Pomier-Layrargues, G., Kirsch, R., Pollett, A., Beaton, M., Levstik, M., et al. (2012) Discordance in Fibrosis Staging between Liver Biopsy and Transient Elastography Using the FibroScan XL Probe. Journal of Hepatology, 56, 564-570. http://dx.doi.org/10.1016/j.jhep.2011.10.007

[24] Wu, J. and Danielsson, A. (1995) Detection of Hepatic Fibrogenesis: A Review of Available Techniques. Scandinavian Journal of Gastroenterology, 30, 817-825. http://dx.doi.org/10.3109/00365529509101585

[25] Lebensztejn, D.M., Wierzbicka, A., Socha, P., Pronicki, M., Skiba, E., Werpachowska, I., et al. (2011) Cytokeratin-18 and Hyaluronic Acid Levels Predict Liver Fibrosis in Children with Non-Alcoholic Fatty Liver Disease. Acta Biochimica Polonica, 58, 563-566.

[26] Schaffler, A., Scholmerich, J. and Buchler, C. (2005) Mechanisms of Disease: Adipocytokines and Visceral Adipose Tissue-Emerging Role in Nonalcoholic Fatty Liver Disease. Nature Reviews Gastroenterology and Hepatology, 2, 273-280. http://dx.doi.org/10.1038/ncpgasthep0186

[27] Harrison, S.A., Kadakia, S., Lang, K.A. and Schenker, S. (2002) Nonalcoholic Steatohepatitis: What We Know in the New Millennium. The American Journal of Gastroenterology, 97, 2714-2724.

[28] Saadeh, S., Cammell, G., Carey, W.D., Younossi, Z., Barnes, D. and Easley, K. (2001) The Role of Liver Biopsy in Chronic Hepatitis C. Hepatology, 33, 196-200. http://dx.doi.org/10.1053/jhep.2001.20534

[29] Poynard, T., Ratziu, V. and Bedossa, P. (2000) Appropriateness of Liver Biopsy. Canadian Journal of Gastroenterology, 14, 543-548.

[30] Schaffler, A., Scholmerich, J. and Buchler, C. (2005) Mechanisms of Disease: Adipocytokines and Visceral Adipose Tissue-Emerging Role in Nonalcoholic Fatty Liver Disease. Nature Reviews Gastroenterology and Hepatology, 2, 273-280. http://dx.doi.org/10.1038/ncpgasthep0186

[31] Harrison, S.A., Kadakia, S., Lang, K.A. and Schenker S. (2002) Nonalcoholic Steatohepatitis: What We Know in the New Millennium. The American Journal of Gastroenterology, 97, 2714-2724.

[32] de Ledinghen, V., Wong, V.W., Vergniol, J., Wong, G.L., Foucher, J., Chu, S.H., et al. (2012) Diagnosis of Liver Fibrosis and Cirrhosis Using Liver Stiffness Measurement: Comparison between M and XL Probe of FibroScan ${ }^{\circledR}$. Journal of Hepatology, 56, 833-839. http://dx.doi.org/10.1016/j.jhep.2011.10.017 